\title{
Quasiparticle picture of high temperature superconductors in the frame of Fermi liquid with the fermion condensate
}

\author{
M.Ya. Amusia ${ }^{a, b}$ and V.R. Shaginyan ${ }^{a, c} *$ \\ ${ }^{a}$ The Racah Institute of Physics, the Hebrew University, Jerusalem 91904, Israel; \\ ${ }^{b}$ Physical-Technical Institute, Russian Academy of Sciences, 194021 St. Petersburg, Russia; \\ c Petersburg Nuclear Physics Institute, Russian Academy of Sciences, Gatchina, 188350, Russia
}

\begin{abstract}
A model of a Fermi liquid with the fermion condensate (FC) is applied to the consideration of quasiparticle excitations in high temperature superconductors, in their superconducting and normal states. Within our model the appearance of the fermion condensate presents a quantum phase transition, that separates the regions of normal and strongly correlated electron liquids. Beyond the phase transition point the quasiparticle system is divided into two subsystems, one containing normal quasiparticles and the other — fermion condensate localized at the Fermi surface and characterized by almost dispersionless single-particle excitations. In the superconducting state the quasiparticle dispersion in systems with $\mathrm{FC}$ can be presented by two straight lines, characterized by effective masses $M_{F C}^{*}$ and $M_{L}^{*}$, respectively, and intersecting near the binding energy which is of the order of the superconducting gap. This same quasiparticle picture persists in the normal state, thus manifesting itself over a wide range of temperatures as new energy scales. Arguments are presented that fermion systems with FC have features of a "quantum protectorate" [1.2].
\end{abstract}

PACS numbers: 71.27.+a, 74.20.Fg, 74.25.Jb

Typeset using REVTEX

*E-mail: vrshag@thd.pnpi.spb.ru 


\section{INTRODUCTION}

The single-particle excitations in ordinary Fermi liquids, e.g., electron liquid of metals, and their energy scales, define the major part of their low temperature properties. High temperature superconductors (HTS) are characterized by a number of striking features. Among them are extremely high transition temperatures, $T_{c}$, and the linear dependence of the resistivity on temperature at $T>T_{c}$. The former behavior has been related to the existence of the only one relevant energy scale, that is the temperature $T$, which leads to a central conclusion of the Marginal Fermi Liquid (MFL) that the one-particle self energy depends only on temperature and frequency, and not on momentum [3]. Such a behavior demonstrates that contributions from phonons excitations, collective states, or impurities to the self energy are inessential. All this permits to introduce the notion of a "quantum protectorate", as a state of a system with so strong correlations that these conventional effects are inessential [1,2]. On the other hand, recent discovery of a new energy scale for quasiparticle dispersion in superconducting and normal states of $\mathrm{Bi}_{2} \mathrm{Sr}_{2} \mathrm{CaCu}_{2} \mathrm{O}_{8+\delta}$, 4, 5] can bring new insight to physics of HTS, imposing serious constraints upon possible theories of HTS. The newly discovered additional energy scale manifests itself as a break in the quasiparticle dispersion near $(50-70)$ meV, which results in a drastic change of the quasiparticle velocity [4] [ Such a behavior is qualitatively different from what one could expect in a normal Fermi liquid. Moreover, this behavior can hardly be understood in the frames of either MFL theory or the quantum protectorate since there are no additional energy scales in these theories [2, 3]. One could suggest that this observed strong self-energy effect, leading to the new energy scale, is due to the electron coupling with collective excitations. But in that case one has to give up the quantum protectorate idea, which would contradict observations [1,2].

The aim of our paper is to show that without giving up the quantum protectorate idea the energy scale for quasiparticle dispersion can be naturally explained within the model of correlated electron liquid with FC. In Sec. 2, we review the general features of Fermi systems with the FC, showing that an electron liquid of low density inevitably undergoes the fermion condensation quantum phase transition (FCQPT). In Sec. 3 we consider the superconducting state, which take place in the presence of the FC and describe the quasiparticle dispersion and lineshape. Finally, in Sec. 4, we summarize our main results.

\section{THE GENERAL FEATURES OF ELECTRON LIQUID WITH FC}

To describe a correlated electron liquid a conventional way can be used, assuming that the correlated regime is connected to the noninteracting Fermi gas by adiabatic continuity in the same way as in the framework of the Landau normal Fermi liquid theory [7]. But a question exists whether this is possible at all. Most likely, the answer is negative. Therefore, we direct our attention to a 
model, in the frame of which a strongly correlated electron liquid is separated from conventional Fermi liquid by a phase transition related to the onset of FC [8,9].

Let us start by considering the key points of the FC theory. FC is a new solution of the Fermi liquid theory equations [7] for the quasiparticle distribution function $n(p, T)$

$$
\frac{\delta(F-\mu N)}{\delta n(p, T)}=\varepsilon(p, T)-\mu(T)-T \ln \frac{1-n(p, T)}{n(p, T)}=0
$$

which depends on the momentum $p$ and temperature $T$. Here $F$ is the free energy, and $\mu$ is the chemical potential, while

$$
\varepsilon(p, T)=\frac{\delta E[n(p)]}{\delta n(p, T)},
$$

is the quasiparticle energy. This energy is a functional of $n(p, T)$ just like the total energy $E[n(p)]$ and the other thermodynamic functions. Eq. (1) is usually presented as the Fermi-Dirac distribution,

$$
n(p, T)=\left\{1+\exp \left[\frac{(\varepsilon(p, T)-\mu)}{T}\right]\right\}^{-1} .
$$

At $T \rightarrow 0$ one gets from Eqs. (1), (3) the standard solution $n_{F}(p, T \rightarrow 0) \rightarrow \theta\left(p_{F}-p\right)$, with $\varepsilon\left(p \simeq p_{F}\right)-\mu=p_{F}\left(p-p_{F}\right) / M_{L}^{*}$, where $p_{F}$ is the Fermi momentum, and $M_{L}^{*}$ is the Landau effective mass [7],

$$
\frac{1}{M_{L}^{*}}=\left.\frac{1}{p} \frac{d \varepsilon(p, T=0)}{d p}\right|_{p=p_{F}}
$$

It is implied that $M_{L}^{*}$ is positive and finite at the Fermi momentum $p_{F}$. As a result, the $T$-dependent corrections to $M_{L}^{*}$, to the quasiparticle energy $\varepsilon(p)$, and other quantities, start with $T^{2}$-terms. But this solution is not the only one possible. There exist "anomalous" solutions of Eq. (1) associated with the so-called fermion condensation [8,10]. Being continuous and satisfying the inequality $0<n(p)<1$ within some region in $p$, such solutions $n(p)$ admit a finite limit for the logarithm in Eq. (1) at $T \rightarrow 0$ yielding,

$$
\varepsilon(p)=\frac{\delta E[n(p)]}{\delta n(p)}=\mu ; p_{i} \leq p \leq p_{f} .
$$

At $T=0$ Eq. (5) determines the FC quantum phase transition (FCQPT), possessing solutions at some $r_{s}=r_{F C}$ as soon as the effective inter-electron interaction becomes sufficiently strong [11]. In a simple electron liquid, the effective inter-electron interaction is proportional to the dimensionless average interparticle distance $r_{s} \sim r_{0} / a_{B}$, with $r_{0} \sim 1 / p_{F}$ being the average distance, and $a_{B}$ is the Bohr radius. Equation (5) leads to the minimal value of $E$ as a functional of $n(p)$ when in system under consideration a strong rearrangement of the single particle spectra can take place. We see from Eq. (5) that the occupation numbers $n(p)$ become variational parameters: the solution $n(p)$ takes 
place if the energy $E$ can be lowered by alteration of the occupation numbers. Thus, within the region $p_{i}<p<p_{f}$, the solution $n(p)=n_{F}(p)+\delta n(p)$ deviates from the Fermi step function $n_{F}(p)$ in such a way that the energy $\varepsilon(p)$ stays constant while outside this region $n(p)$ coincides with $n_{F}(p)$. It follows from the above consideration that the superconductivity order parameter $\kappa(\mathbf{p})=\sqrt{n(\mathbf{p})(1-n(\mathbf{p}))}$ has a nonzero value over the region occupied by FC. The superconducting gap $\Delta(\mathbf{p})$ being linear in the coupling constant of the particle-particle interaction $V_{p p}$ gives rise to the high value of $T_{c}$ because one has $2 T_{c} \simeq \Delta$ within the standard Bardeen-Cooper-Schrieffer (BCS) theory [12]. On the other hand, even at $T=0, \Delta$ can vanish, provided $V_{p p}$ is repulsive or absent. Then, as it is seen from Eq. (5), the Landau quasiparticle system becomes separated into two subsystems. The first contains the Landau quasiparticles, while the second, related to FC, is localized at the Fermi surface and formed by dispersionless quasiparticles. As a result, the standard Kohn-Sham scheme for the single particle equations is no longer valid beyond the point of the FC phase transition [13]. Such a behavior of systems with FC is clearly different from what one expects from the well known local density calculations. Therefore these calculations are hardly applicable to describe systems with FC. It is also seen from Eq. (5) that a system with FC has a well-defined Fermi surface.

Let us assume that FC has just taken place, that is $p_{i} \rightarrow p_{f} \rightarrow p_{F}$, and the deviation $\delta n(p)$ is small. Expanding functional $E[n(p)]$ in Taylor's series with respect to $\delta n(p)$ and retaining the leading terms, one obtains from Eq. (5),

$$
\mu=\varepsilon(\mathbf{p})=\varepsilon_{0}(\mathbf{p})+\int F_{L}\left(\mathbf{p}, \mathbf{p}_{1}\right) \delta n\left(\mathbf{p}_{1}\right) \frac{d \mathbf{p}_{1}}{(2 \pi)^{2}} ; p_{i} \leq p \leq p_{f},
$$

where $F_{L}\left(\mathbf{p}, \mathbf{p}_{1}\right)=\delta^{2} E / \delta n(\mathbf{p}) \delta n\left(\mathbf{p}_{1}\right)$ is the Landau interaction. Both the Landau interaction and the single-particle energy $\varepsilon_{0}(p)$ are calculated at $n(p)=n_{F}(p)$. It is seen from Eq. (6) that the FC quasiparticles forms a collective state, since their energies are defined by the macroscopical number of quasiparticles within the region $p_{i}-p_{f}$, and vice versa. The shape of the spectra is not effected by the Landau interaction, which, generally speaking, depends on the system's properties, including the collective states, impurities, etc. The only thing defined by the interaction is the width of the region $p_{i}-p_{f}$, provided the interaction is sufficiently strong to produce the $\mathrm{FC}$ phase transition at all. Thus, we can conclude that the spectra related to FC are of universal form, being dependent, as we will see below, mainly on temperature $T$, if $T>T_{c}$, or on the superconducting gap at $T<T_{c}$.

According to Eq. (1), the single-particle excitations within the interval $p_{i}-p_{f}$ have at $T_{c} \leq T \ll$ $T_{f}$ the shape $\varepsilon(p, T)$ linear in $\mathrm{T}$ 12,14, which can be simplified at the Fermi level,

$$
\varepsilon(p, T)-\mu(T)=\left.T \ln \frac{1-n(p)}{n(p)} \simeq T \frac{1-2 n(p)}{n(p)}\right|_{p \simeq p_{F}} .
$$

$T_{f}$ is the temperature, above which FC effects become insignificant [12],

$$
\frac{T_{f}}{\varepsilon_{F}} \sim \frac{p_{f}^{2}-p_{i}^{2}}{2 M \varepsilon_{F}} \sim \frac{\Omega_{F C}}{\Omega_{F}} .
$$


Here $\Omega_{F C}$ is the FC volume, $\varepsilon_{F}$ is the Fermi energy, and $\Omega_{F}$ is the volume of the Fermi sphere. We note that at $T_{c} \leq T \ll T_{f}$ the occupation numbers $n(p)$ are approximately independent of $T$, being given by Eq. (5). One can imagine that at these temperatures dispersionless plateau $\varepsilon(p)=\mu$ given by Eq. (5) is slightly turned counter-clockwise about $\mu$. As a result, the plateau is just a little tilted and rounded off at the end points. According to Eq. (7) the effective mass $M_{F C}^{*}$ related to FC is given by,

$$
\frac{p_{F}}{M_{F C}^{*}} \simeq \frac{4 T}{p_{f}-p_{i}} .
$$

To obtain Eq. (9) an approximation for the derivative $d n(p) / d p \simeq-1 /\left(p_{f}-p_{i}\right)$ was used. Having in mind that $p_{f}-p_{i} \ll p_{F}$, and using (8) and (9) the following estimates for the effective mass $M_{F C}^{*}$ are obtained,

$$
\frac{M_{F C}^{*}}{M_{0}} \sim \frac{N(0)}{N_{0}(0)} \sim \frac{T_{f}}{T}
$$

Eqs. (9) and (10) show the temperature dependence of $M_{F C}^{*}$. In (10) $M_{0}$ denotes the bare electron mass, $N_{0}(0)$ is the density of states of noninteracting electron gas, and $N(0)$ is the density of states at the Fermi level. Multiplying both sides of Eq. (9) by $p_{f}-p_{i}$ we obtain the energy scale $E_{0}$ separating the slow dispersing low energy part, related to the effective mass $M_{F C}^{*}$, from the faster dispersing relatively high energy part, defined by the effective mass $M_{L}^{*}$ [15],

$$
E_{0} \simeq 4 T
$$

It is seen from Eq. (11) that the scale $E_{0}$ does not depend on the condensate volume. The single particle excitations are defined according to Eqs. (7) and (9) by the temperature and by $n(p)$, given by Eq. (5). Thus, we are led to the conclusion that the one-electron spectrum is negligible disturbed by thermal excitations, impurities, etc, so that one observes the features of the quantum protectorate.

It is seen from Eq. (5) that at the point of FC phase transition $p_{f} \rightarrow p_{i} \rightarrow p_{F}, M_{F C}^{*}$ and the density of states, as it follows from Eqs. (5), (10), tend to infinity. One can conclude that at $T=0$ the beginning of the FC phase transition is connected to the absolute growth of $M_{L}^{*}$. It is essential to have in mind, that the onset of the charge density wave instability in a many-electron system, such as electron liquid, which takes place as soon as the effective inter electron constant reaches its critical value $r_{s}=r_{c d w}$ [16], is preceded by the unlimited growth of the effective mass. Therefore, the FC takes place before the onset of the charge density wave. Hence, at $T=0$, when $r_{s}$ reaches its critical value $r_{F C}<r_{c d w}$, the FCQPT inevitably takes place. Thus, the formation of the FC can be thought as a general property of an electron liquid of low density, rather then an uncommon and anomalous solution of Eq. (1) [11]. Beyond the phase transition into the FC the condensate volume is proportional to $\left(r_{s}-r_{F C}\right)$ as well as $T_{f} / \varepsilon_{F} \sim\left(r_{s}-r_{F C}\right)$ at least when $\left(r_{s}-r_{F C}\right) / r_{F C} \ll 1$. Note, that such a behavior is in accordance with the general properties of 
second order phase transitions. Therefore, we can accept a model relating systems with FC to HTS compounds, assuming that the effective coupling constant $r_{s}$ increases with decreasing doping, exceeding its critical value $r_{F C}$ at the levels corresponding to optimal doped samples. We remark, that this critical value $r_{F C}$ corresponds to the $r_{s}$ values of slightly overdoped samples [11]. On the other hand, there exist charge density waves or strong fluctuations of charge ordering in underdoped HTS [17]. As the result, our quite natural model suggests that both quantities, $T_{f}$ and condensate volume $\Omega_{F C}$, increase with decrease of doping. Thus, these values are higher in underdoped samples as compared to overdoped ones provided $r_{s}$ meets the mentioned above conditions. According to experimental facts the large density of states at the Fermi level reaches its maximum in the vicinity of the Hove singularities, that is around the point $(\pi, 0)$ of the Brillouin zone, or $\bar{M}$, in HTS compounds. The density of states reaches its minimum value at the intersection of the so called nodal direction of the Brillouin zone with the Fermi surface (see, e.g., [18]). The FC sets in around the van Hove singularities [14], causing, according to Eqs. (9) and (10), large density of states and large value of the difference $\left(p_{f}-p_{i}\right)$ at the point $\bar{M}$. Then, the volume $\Omega_{F C}$ and difference $\left(p_{f}-p_{i}\right)$ start to depend on the point of the Fermi surface, say, on the angle $\phi$ along the Fermi surface, which we count from the point $\bar{M}$ to the point at which the density of states reaches its minimum value. Nonetheless, as it is seen from Eq. (11), $E_{0}$ remains constant, being independent of the angle. This differs essentially from the case for the effective mass $M_{F C}^{*}$, that can strongly depend upon the angle via the difference $\left(p_{f}(\phi)-p_{i}(\phi)\right)$, as it is seen from Eq. (9). It is pertinent to note that outside the $\mathrm{FC}$ region the single particle spectrum is negligible affected by the temperature, being defined by $M_{L}^{*}$, (see Eq. (4)), however calculated at $p \leq p_{i}$ instead of at $p=p_{F}$. Thus, we come to the conclusion that a system with $\mathrm{FC}$ is characterized by two effective masses: $M_{F C}^{*}$ that is related to the single particle spectrum at lower energy scale, and $M_{L}^{*}$ describing the spectrum at higher energy scale. These two effective masses manifest itself as a break in the quasiparticle dispersion, which can be approximated by two straight lines intersecting at the energy $E_{0}$. This break takes place at temperatures $T_{c} \leq T \ll T_{f}$ in accordance with the experimental findings [4, and, as we will see, at $T \leq T_{c}$ corresponding to the experimental facts [4, 5], when the superconducting state is based on the FC state. As to the quasiparticle formalism, it is applicable to this problem since the width $\gamma$ of single particle excitations is not large compared to their energy being proportional $\gamma \sim T$ at $T>T_{c}$ [12]. The lineshape can be approximated by a simple Lorentzian [15], being in accordance with experimental data obtained from scans at a constant binding energy [6]. Then, FC serves as a stimulating source of new phase transitions which lift the degeneration of the spectrum. For example, FC can generate the spin density wave, or antiferromagnetic phase transition, thus leading to a whole variety of the system's properties. Then, the onset of the charge density wave is preceded by the FCQPT, and both of these phases can coexist at the sufficiently low density when $r_{s} \geq r_{c d w}$. The simple consideration presented above explains extremely large variety of HTS properties. We have seen above that the superconductivity is strongly aided by the FC, because both of the phases 
are characterized by the same order parameter. As a result, the superconductivity, removing the spectrum degeneration, "wins the competition" with the other phase transitions up to the critical temperature $T_{c}$. We turn now to a consideration of quasiparticle dispersions at $T \leq T_{c}$.

\section{QUASIPARTICLE DISPERSIONS AT $T \leq T_{C}$}

Let us discuss the origin of two effective masses $M_{L}^{*}$ and $M_{F C}^{*}$ in the superconducting state resulting in nontrivial quasiparticle dispersion and in alteration of the quasiparticle velocity. As we will see our results are in a reasonably good agreement with experimental data [46]. To simplify the discussion let us put $T=0$. The ground state energy $E_{g s}$ of a system in the superconducting state is given by the BSC theory formula

$$
E_{g s}[\kappa(\mathbf{p})]=E[n(\mathbf{p})]+E_{s c}[\kappa(\mathbf{p})]
$$

where the occupation numbers $n(\mathbf{p})$ are connected to the order parameter,

$$
n(\mathbf{p})=v^{2}(\mathbf{p}) ; \kappa(\mathbf{p})=v(\mathbf{p}) \sqrt{\left(1-v^{2}(\mathbf{p})\right)} .
$$

The second term $E_{s c}\left[\kappa_{p}\right]$ on the right hand side of Eq. (12) is defined by the superconducting contribution which in the simplest case of the weak coupling regime is of the form,

$$
E_{s c}\left[\kappa_{p}\right]=\int V_{p p}\left(\mathbf{p}_{1}, \mathbf{p}_{2}\right) \kappa\left(\mathbf{p}_{1}\right) \kappa^{*}\left(\mathbf{p}_{2}\right) \frac{d \mathbf{p}_{1} d \mathbf{p}_{2}}{(2 \pi)^{4}}
$$

Consider a two-dimensional electron liquid on a simple square lattice which is in the superconducting state with d-wave symmetry of the order parameter $\kappa(\mathbf{p})$. In such a case, the long-range component in momentum space of particle-particle interaction $V_{p p}$ is repulsive, and the short-range component is relatively dominant and attractive at small momenta [19]. Then the short-range component can be taken as the first approximation to $V_{p p}(q) \simeq-V_{2} \delta(q)$. The $\mathrm{FC}$ arises near the Van Hove singularities, causing, as it follows from Eq. (10), large density of states at these points [14]. Hence, the different regions with the maximal value $\Delta_{1}$ of the gap $\Delta$ and the maximal density states overlap slightly 10,19,20. Varying $E_{g s}$ given by Eq. (12) with respect to $\kappa(\mathbf{p})$ one finds,

$$
\varepsilon(\mathbf{p})-\mu=\Delta(\mathbf{p}) \frac{1-2 v^{2}(\mathbf{p})}{2 \kappa(\mathbf{p})} .
$$

Here $\varepsilon(\mathbf{p})$ is defined by Eq. (2), and,

$$
\Delta(\mathbf{p})=-\int V_{p p}\left(\mathbf{p}, \mathbf{p}_{1}\right) \sqrt{n\left(\mathbf{p}_{1}\right)\left(1-n\left(\mathbf{p}_{1}\right)\right)} \frac{d \mathbf{p}_{1}}{4 \pi^{2}} .
$$

A few remarks are in order at this point. If $V_{2} \rightarrow 0$, then $\Delta(\mathbf{p}) \rightarrow 0$, and Eq. (15) reduces to the equation, 


$$
\varepsilon(\mathbf{p})-\mu=0, \text { if } 0<n(\mathbf{p})<1 ; \kappa(\mathbf{p}) \neq 0,
$$

presenting FC solutions, defined by Eq. (5) [12, [13]. Thus, we come to the conclusion that the function $\kappa(\mathbf{p})$ is defined by Eq. (5). While corrections to this function due to the pairing interaction, being small, are of the order of $V_{p p} / F_{L}$, because interaction $V_{p p}$ is obviously weak as compared to the Landau interaction $F_{L}$. We note again remarkable peculiarity of the $\mathrm{FC}$ phase transition at $T=0$ : this transition is related to spontaneous breaking of gauge symmetry, when the superconductivity order parameter $\kappa(\mathbf{p})$ has a nonzero value over the region occupied by the fermion condensate, while $\Delta(\mathbf{p})$ vanishes provided $V_{p p}=0$ [12, 13]. We can conclude that the transition temperature of the FC phase transition is zero because it is proportional to the gap, as it must be in the standard theory of superconductivity. Therefore, the FC phase transition is a quantum phase transition, while, at temperatures $T \ll T_{f}$, the properties of considered many-electron system, such as its single particle spectra, occupation numbers and etc. are strongly influenced by the "shadow" of FCQPT as it is seen from Eqs. (9-11).

If $V_{p p}$ is nonzero but small as compared to $F_{L}$ and attractive, the gap $\Delta$ is given by Eq. (16), with $n(\mathbf{p})$ and $\kappa(\mathbf{p})$ being determined by Eq. (5). Therefore, as it is seen from Eq. (16), the gap is linear in the coupling constant of the particle-particle interaction $V_{2}$, which leads to high values of both $\Delta_{1}$ and $T_{c}\left[\mathbb{8}\right.$. Taking into account the $\delta$-function shape of the attractive component of $V_{p p}$, we have from Eq. (16) simple estimations for the maximum value of the gap: $2 \Delta_{1} \simeq V_{2}$. Since the order parameter $\kappa(\mathbf{p})$ is defined by Eq. (5), that is determined by the interaction $F_{L}$, the shape of the gap, including the location of its nodes is robust being resistant to scattering upon impurities. We can again conclude that such features resemble a quantum protectorate. Generally speaking, the state of the quantum protectorate is preserved by the FCQPT. As soon as the coupling constant $V_{2}$ becomes finite (although remains small), the plateau $\varepsilon(\mathbf{p})-\mu=0$ is slightly tilted and rounded off at the end points, that is the effective mass $M_{F L}^{*}$ becomes finite. To calculate $M_{F L}^{*}$, we differentiate the both parts of Eq. (15) with respect to the momentum $p$ and obtain the following relations,

$$
\left.\frac{p_{F}}{M_{F L}^{*}} \simeq \frac{\Delta_{1}}{4 \kappa(\mathbf{p})} \frac{1}{p_{f}-p_{i}} \simeq \frac{2 \Delta_{1}}{p_{f}-p_{i}}\right|_{p \simeq p_{F}} .
$$

Deriving Eq. (18) we took into account that the gap achieves its maximum value $\Delta_{1}$ at the Fermi level, and $\kappa\left(p \simeq p_{F}\right) \simeq 1 / 2$. We use the above approximation for the derivative $d n / d p$ and Eq. (13) to calculate the derivative $d\left(v^{2}\right) / d p$ :

$$
\frac{d\left(v^{2}(p)\right)}{d p} \simeq-\frac{1}{\left(p_{f}-p_{i}\right)} .
$$

Now, one can conclude directly from Eq. (18), that the following relation is valid 15

$$
E_{0} \simeq \frac{\left(p_{f}-p_{i}\right) p_{F}}{M_{F C}^{*}} \simeq 2 \Delta_{1} .
$$


It is seen from Eq. (19) that again, this time at $T=0$, the quasiparticle dispersion can be presented by two straight lines characterized by two effective masses, $M_{F C}^{*}$ and $M_{L}^{*}$, respectively, and intersecting near the binding energy $E_{0} \simeq 2 \Delta_{1}$. Evaluations of the effective mass $M_{F L}^{*}$ at $T \rightarrow T_{c}$ is straightforward and similar to the presented above. It is important that at finite temperatures we have to replace Eq. (13) by another equation of the BCS theory, namely by

$$
v^{2}(\mathbf{p})=\frac{n(\mathbf{p})-f(\mathbf{p})}{1-2 f(\mathbf{p})}
$$

where,

$$
f(\mathbf{p})=\frac{1}{1+\exp [E(\mathbf{p}) / T]} ; \quad E(\mathbf{p})=\sqrt{(\varepsilon(\mathbf{p})-\mu)^{2}+\Delta^{2}(\mathbf{p})} .
$$

After performing some straightforward algebraic transformations and taking into account that the function $f(\mathbf{p})$ reaches its maximum at the Fermi level, while $E(\mathbf{p}) \ll T$, we obtain instead of Eq. (19) the following equations

$$
\frac{d\left(v^{2}(p)\right)}{d p} \simeq-\frac{1}{\left(p_{f}-p_{i}\right)(1-2 f(\mathbf{p}))} \simeq-\left.\frac{2 T}{E(p)\left(p_{f}-p_{i}\right)}\right|_{T \rightarrow T_{c}} .
$$

Deriving Eq. (23) we use the former approximation for $d n / d p$ and have in mind that at $T \ll T_{f}$ the occupation numbers are temperature independent and defined by Eq. (5). Differentiating Eq. (15) with respect to the momentum $p$ and taking into account Eq. (23), we estimate the effective mass as

$$
M_{F L}^{*} \simeq \frac{p_{F}\left(p_{f}-p_{i}\right)}{4 T} .
$$

As the result, we obtain from Eq. (24) an estimation for the energy scale,

$$
E_{0} \simeq \frac{\left(p_{f}-p_{i}\right) p_{F}}{M_{F C}^{*}} \simeq 4 T .
$$

Comparing Eq. (20) with (25) and bearing in mind that $2 T_{c} \simeq \Delta_{1}$ we conclude that both the effective mass $M_{F C}^{*}$ and the energy scale $E_{0}$ are approximately temperature independent at $T \leq T_{c}$, while Eqs. (24) and (25) match Eqs. (9) and (11) at $T=T_{c}$, as one should expect.

The break separating the faster dispersing high energy part, related to mass $M_{L}^{*}$, from the slower dispersing low energy part defined by $M_{F C}^{*}$, is likely to be more pronounced in underdoped samples. That is at least because of the rise of the condensate volume $\Omega_{F C}$, leading to the growth of $M_{F C}^{*}$ as it follows from Eqs. (9) and (18). We remind that according our model the condensate volume $\Omega_{F C}$ is growing with underdoping. It follows from Eqs. (9) and (18), that as one moves along the Fermi surface from the nodal direction towards the point $\bar{M}$, that is from minimal value of $\Omega_{F C}(\phi) \sim$ $\left(p_{f}^{2}(\phi)-p_{i}^{2}(\phi)\right)$ towards the maximal one, the ratio $M_{F C}^{*} / M_{L}^{*}$ grows in magnitude, transforming the dispersion kink into a distinct break at the point $\bar{M}$, or at the gap maximum $\Delta_{1}$ point. Thus, as it follows from Eqs. $(11)$ and $(20)$ at $T \ll T_{f}$ there exists a new energy scale defined by $E_{0}$, with 
$E_{0} \simeq 2 \Delta_{1}$ at $T \leq T_{c}$ and $E_{0} \simeq 4 T$ at $T_{c} \leq T$. These results are in good agreement with the experimental facts which show that at $T \leq T_{c}$ as one moves towards $\bar{M}$ the dispersion kink grows into the break separating the faster dispersing high energy part of the single-particle spectrum from the slower dispersing low energy part with a break in the slope near $50 \mathrm{meV}$ [4], or near $70 \mathrm{meV}$ [5]. This effect is enhanced in underdoped samples, and appears to persist at $T_{c} \leq T$ [4].

Let us briefly discuss the lineshape of a quasiparticle peak obtained from scans at a constant binding energy $\omega$ [6], and at a constant momentum $q$, see e.g. [18]. We recall that the lineshape $L$ of a quasiparticle peak can be presented as a function of two variables: $L(q, \omega)$. Then, the scans at constant binding energy is given by the function $L\left(q, \omega=\omega_{0}\right)$, with $\omega_{0}$ is the binding energy of the quasiparticle. Accordingly, $L\left(q=q_{0}, \omega\right)$ presents the lineshape obtained from scans at the fixed momentum $q_{0}$ corresponding to the quasiparticle momentum. In order to consider the width $\gamma$ of a quasiparticle peak, the special form of the quasiparticle dispersion characterized by the two effective masses should be taken into consideration. On the other hand, scans at the constant energy reveal well defined single-particle excitations with the width $\gamma \sim T$ at the Fermi level even at the point $\bar{M}$ [6]. Considering $\gamma$ related to the lineshape $L\left(q, \omega=\omega_{0}\right)$, provided $\omega_{0} \leq E_{0}$, we can take into account only quasiparticles with the effective mass $M_{F C}^{*}$, which can be large but finite. We can do it because only quasiparticles with the energies less then $E_{0}$ contribute to the width of a quasiparticle with the energy $\omega_{0}$. Such a picture resembles the normal Fermi liquid presented by quasiparticles with the effective mass $M_{F C}^{*}$. The only difference is that now the effective mass, as it follows from Eq. (9), depends on the temperature. As the result, we are dealing with well-defined excitations of the width $\gamma \sim T$ 12]

$$
\gamma \sim \frac{\left(M_{F C}^{*}\right)^{3} T^{2}}{\epsilon^{2}} \sim \frac{\left(M_{F C}^{*}\right)^{3} T^{2}}{\left(M_{F C}^{*}\right)^{2}} \sim T \frac{T_{f}}{\varepsilon_{F}} .
$$

Here $\epsilon$ is the dielectric constant, which is proportional to the effective mass $M_{F C}^{*}$, the latter being inversely proportional to $T$, see Eq. (9). This result is in good agreement with the experimental findings cited above [6]. Dealing with scans at constant $q$, which correspond to the lineshape function $L\left(q=q_{0}, \omega\right)$, we have to consider the contribution coming from quasiparticles with the mass $M_{L}^{*}$ as well, because now there are no limits on the energy of quasiparticles contributing to the width $\gamma$. In view of the fact that the contribution of these excitations is enhanced by the presence of $\mathrm{FC}$, and these excitations start to contribute to the lineshape at energies $\omega \geq E_{0}$, one can conclude that the peak inevitably has a broadening which can hardly be interpreted as standard width obtained from scans at a constant binding energy. On the other hand, one may follow the procedure suggested in [5], using the Kramers-Krönig transformation to construct the imaginary part of the self-energy starting with the real one. As a result, the lineshape $L\left(q=q_{0}, \omega\right)$ of the quasiparticle peak as a function of the binding energy $\omega$ possesses a complex peak-dip-hump structure [5] directly defined by the existence of the two effective masses $M_{F C}^{*}$ and $M_{L}^{*}$ [15]. 


\section{CONCLUDING REMARKS}

We have discussed the model of a strongly correlated electron liquid based on the FCQPT and applied it to high-temperature superconductors. The FCQPT plays the role of a boundary separating the region of a strongly interacting normal electron liquid from the region of a strongly correlated electron liquid. It is important to have in mind, that the onset of the charge density wave instability in a many-electron system, which takes place as soon as the effective inter-electron constant reaches its critical value $r_{s}=r_{c d w}$, is preceded by the FCQPT. Hence at $T=0$, when $r_{s}$ reaches its

critical value $r_{F C}<r_{c d w}$, the FCQPT inevitably takes place. Thus, the FC can be thought as a general property of an electron liquid of the low density rather then a unique phenomenon. We have shown that the quasiparticle dispersion in systems with FC can be represented by two straight lines characterized by the respective effective masses $M_{F C}^{*}$ and $M_{L}^{*}$. At $T<T_{c}$, these lines intersect near the point $E_{0} \sim 2 \Delta_{1}$, while above $T_{c}$, we have $E_{0} \sim 4 T$. It is argued that this strong change of the quasiparticle dispersion at $E_{0}$ can be enhanced in underdoped samples because of strengthening the FC influence. The single-particle excitations and their width $\gamma$ are also studied. Well-defined excitations with $\gamma \sim T$ exist at the Fermi level even in the normal state. This result is in line with the experimental findings determined from the scans at constant binding energies $\omega$. We have discussed also the lineshape obtained from scans at a constant momentum $q$. In this case, the special form of the quasiparticle dispersion should be taken into consideration. As the result, the lineshape of the quasiparticle peak as a function of the binding energy $\omega$ possesses a complex peak-dip-hump structure directly defined by the existence of the two effective masses $M_{F C}^{*}$ and $M_{L}^{*}$. We have also presented arguments that fermion systems with $\mathrm{FC}$ have features of the quantum protectorate, being separated from the normal Fermi liquid by the FC quantum phase transition.

\section{ACKNOWLEDGEMENT}

V.R.S. thanks the Racah Institute of Physics at the Hebrew University of Jerusalem where part of this work has been done for hospitality. This research was supported in part by the Russian Foundation for Basic Research under Grant No. 01-02-17189. 


\section{REFERENCES}

[1] R.B. Laughlin and D. Pines, PNAS, Jan. 1, 2000.

[2] P.W. Anderson, cond-mat/0007185; cond-mat/0007287.

[3] C.M. Varma et. al., Phys. Rev. Lett. 63, 1996 (1989); C.M. Varma it et. al., Phys. Rev. Lett. 64, 497 (1990); E. Abrahams and C.M. Varma, cond-mat/0003135.

[4] P.V. Bogdanov et al., Phys. Rev. Lett. 85, 2581 (2000).

[5] A. Kaminski et al., Phys. Rev. Lett. 86, 1070 (2001).

[6] T. Valla et al., Science 285, 2110 (1999); T. Valla et al., Phys. Rev. Lett. 85, 828 (2000).

[7] L.D. Landau, Sov. Phys. JETP 30, 1058 (1956).

[8] V.A. Khodel and V.R. Shaginyan, JETP Lett. 51, 553 (1990).

[9] G.E. Volovik, JETP Lett. 53, 222 (1991).

[10] V.A. Khodel, V.R. Shaginyan, and V.V. Khodel, Phys. Rep. 249, 1 (1994).

[11] V.A. Khodel, V.R. Shaginyan, and M.V. Zverev, JETP Lett. 65, 253 (1997).

[12] J. Dukelsky et al., Z. Phys. 102, 245 (1997); V.A. Khodel and V.R. Shaginyan, Condensed Matter Theories, 12, 222 (1997).

[13] V.R. Shaginyan, Phys. Lett. A 249, 237 (1998).

[14] V.A. Khodel, J.W. Clark, and V.R. Shaginyan, Solid Stat. Comm. 96, 353 (1995).

[15] M.Ya. Amusia and V.R. Shaginyan, Phys. Lett. A 275, 124 (2000); S.A. Artamonov and V.R. Shaginyan, JETP 92, 287 (2001).

[16] L. Świerkowski, D. Neilson, and J. Szymański, Phys. Rev. Lett. 67, 240 (1991).

[17] G. Grüner, Density Waves in Solids (Addison-Wesley, Reading, MA, 1994).

[18] Z.X. Shen and D. Dessau, Phys. Rep. 253, 1 (1995).

[19] A.A. Abrikosov, Phys. Rev. B 52, R15738 (1995); A.A. Abrikosov, cond-mat/9912394.

[20] M.Ya. Amusia and V.R. Shaginyan, Phys. Lett. A 259, 460 (1999). 